\title{
Semicontinuity of the Solution Mapping to a Parametric Generalized Weak Ky Fan Inequality
}

\author{
Y. D. Xu \\ College of Science, Chongqing University of Posts and Telecommunications, Chongqing 400065, China \\ Correspondence should be addressed to Y. D. Xu; xyd04010241@126.com
}

Received 6 June 2014; Accepted 7 October 2014; Published 3 November 2014

Academic Editor: Allan Peterson

Copyright (C) 2014 Y. D. Xu. This is an open access article distributed under the Creative Commons Attribution License, which permits unrestricted use, distribution, and reproduction in any medium, provided the original work is properly cited.

Under new assumptions, which do not contain any information about the solution set, the upper and lower semicontinuity of the solution mapping to a class of parametric generalized weak Ky Fan inequality are established by using a nonlinear scalarization technique. These results extend and improve the recent ones in the literature. Some examples are given to illustrate our results.

\section{Introduction}

It is well known that the Ky Fan inequality is a very general mathematical model, which embraces the formats of several disciples, as equilibrium problems of economics, game theory, (vector) optimization problems, (vector) variational inequality problems, and so on (see $[1,2])$. In the literature, existence results for various types of Ky Fan inequalities have been investigated intensively; for example, see [3-5] and the references therein. The stability analysis of solution mappings for parametric Ky Fan inequalities is another important topic in the vector optimization theories and applications. Recently, there have been many results on the continuity, especially the lower semicontinuity, of the solution mappings for parametric (generalized) Ky Fan inequalities in the literature; for example, see [6-22], where the (generalized) Ky Fan inequalities are called vector equilibrium problems or generalized systems.

Among many methods for dealing with the lower semicontinuity and continuity of the solution mappings for (generalized) Ky Fan inequalities, the linear scalarization technique is a kind of efficient approach. Recently, Cheng and Zhu [8] investigated the upper semicontinuity and lower semicontinuity of the solution mappings to parametric weak vector variational inequalities in finite-dimension Euclidean spaces by the linear scalarization method. By following the ideas in [8] and based on a theorem of Berge [23] saying that the union of a family of lower semicontinuous set-valued mappings is lower semicontinuous, Gong [9], Chen et al. [13], and $\mathrm{Li}$ et al. [15] have extended the lower semicontinuity results of Cheng and Zhu [8] to parametric generalized weak Ky Fan inequalities under a suitable strict cone-monotonicity assumption. Li and Fang [16], Peng et al. [18], Chen and Huang [19], and Wang and Li [17] have used the linear scalarization approach to improve the lower semicontinuity results in $[9,13,15]$ by weakening the strict cone-monotonicity assumption. However, the key assumption in $[16,18,19]$ involves the information on the solution set.

It is worth noting that the linear scalarization approach to the semicontinuity of solution mappings in $[8,9,13,15-$ 19] requires (generalized) cone-convexity of the objective mappings or strict cone-monotonicity. To avoid using these assumptions, nonlinear scalarization approaches have been applied for discussing the stability analysis in parametric generalized Ky Fan inequalities. Namely, Sach [20] has used some nonlinear scalarization functions (generalized versions of Gerstewizt's function) to discuss the lower semicontinuity of the solution mappings of parametric generalized weak Ky Fan inequalities. Sach and Tuan [21] also applied the functions to discuss some more generalized cases and obtained the upper and lower semicontinuity of the solution mappings to the problems.

However, the results in $[20,21]$ require that the objective mappings of the discussed problems have compact or cone-closed values, since the definition and propositions of the nonlinear functions defined in $[20,21]$ require these 
assumptions. It may restrict its application scope. Furthermore, the assumptions in $[20,21]$ involve information about the solution set. Obviously, it is not reasonable from the practical point of view. In this paper, we are interested in the study of the semicontinuity of the solution mapping to a class of parametric generalized weak Ky Fan inequality with moving cones. To avoid using the assumptions involving the information about the solution set, we establish some new assumptions and obtain the upper and lower semicontinuity of the solution mapping to the parametric generalized weak Ky Fan inequality by using a nonlinear scalarization function defined in [24], which is different from the nonlinear scalarization functions defined in $[20,21]$.

The rest of the paper is organized as follows. In Section 2, we recall some basic concepts and preliminary results. In Section 3, under some new assumptions, which do not involve any information about the solution set, we give some sufficient conditions for the upper and lower semicontinuity of the solution mapping to the parametric generalized weak Ky Fan inequality. Meanwhile, some examples are provided to illustrate our main results.

\section{Preliminaries}

Throughout this paper, let $T$ and $X$ be Hausdorff topological spaces, and let $A_{i}: T \times K \rightrightarrows K, i=0,1$, be set-valued mappings with nonempty values. Let $Y$ be a locally convex topological vector space, let $C: T \times X \times X \rightrightarrows Y$ be a set-valued mapping such that each value of $C$ is a proper, closed, and convex cone with nonempty interior, and let $F: T \times X \times X \rightrightarrows$ $Y$ be a set-valued mapping with nonempty values. For each $t \in T$, we consider the following parametric generalized weak Ky Fan inequality with moving cones:

problem (PGWKFI): find a point $x \in X$ such that $x \in$ $A_{0}(t, x)$ and, for all $\eta \in A_{1}(t, x)$,

$$
F(t, x, \eta) \subset Y \backslash-\operatorname{int} C(t, x, \eta) \text {. }
$$

For each $t \in T$, we denote by $S(t)$ the solution set of problem (PGWKFI). Throughout this paper, we assume that $S(t) \neq \emptyset$ for all $t \in T$. In this paper, we will discuss the upper and lower semicontinuity of the solution mapping $S(\cdot)$ as a set-valued mapping from the set $T$ to $X$.

Suppose that $G: T \rightrightarrows X$ is a set-valued mapping, and $\bar{t} \in T$ is given.

Definition 1 (see [25]). (i) $G$ is called lower semicontinuous (l.s.c) at $\bar{t}$ if and only if, for any open set $V \subseteq X$ with $V \cap G(\bar{\lambda}) \neq$ $\emptyset$, there exists a neighborhood $N(\bar{t})$ of $\bar{t}$ such that $G(t) \cap V \neq \emptyset$, for all $t \in N(\bar{t})$.

(ii) $G$ is called upper semicontinuous (u.s.c) at $\bar{t}$ if and only if, for any open set $V \subseteq X$ with $G(\bar{t}) \subseteq V$, there exists a neighborhood $N(\bar{t})$ of $\bar{t}$ such that $G(t) \subseteq V$, for all $t \in N(\bar{t})$.

We say $G(\cdot)$ is l.s.c (resp., u.s.c) on $T$ if and only if it is l.s.c (resp., u.s.c) at each $\bar{t} \in T . G(\cdot)$ is said to be continuous on $T$ if and only if it is both l.s.c and u.s.c on $T$.
Proposition 2 (see $[25,26])$. (i) $G$ is l.s.c at $\bar{t}$ if and only if, for any net $\left\{t_{\alpha}\right\} \subseteq T$ with $t_{\alpha} \rightarrow \bar{t}$ and any $\bar{x} \in G(\bar{t})$, there exists $a$ net $\left\{x_{\alpha}\right\} \subseteq G\left(t_{\alpha}\right)$ such that $x_{\alpha} \rightarrow \bar{x}$.

(ii) If $G$ has compact values (i.e., $G(t)$ is a compact set for each $t \in T)$, then $G$ is u.s.c at $\bar{t}$ if and only if, for any net $\left\{t_{\alpha}\right\} \subseteq T$ with $t_{\alpha} \rightarrow \bar{t}$ and any $x_{\alpha} \in G\left(t_{\alpha}\right)$, there exist $\bar{x} \in G(\bar{t})$ and $a$ subnet $\left\{x_{\beta}\right\}$ of $\left\{x_{\alpha}\right\}$, such that $x_{\beta} \rightarrow \bar{x}$.

Definition 3 (see [24]). Let $e: T \times X \times X \rightarrow Y$ be a vectorvalued mapping and, for any $(t, x, \eta) \in T \times X \times X, e(t, x, \eta) \in$ int $C(t, x, \eta)$. The nonlinear scalarization function $\xi: T \times X \times$ $X \times Y \rightarrow \mathbb{R}$ is defined by

$$
\xi(t, x, \eta ; z)=\min \{\lambda \in \mathbb{R}: z \in \lambda e(t, x, \eta)-C(t, x, \eta)\} .
$$

Proposition 4 (see [24]). The function $\xi$ defined in Definition 3 satisfies the following propositions:

(i) $\xi(t, x, \eta ; z)<r \Leftrightarrow z \in \operatorname{re}(t, x, \eta)-\operatorname{int} C(t, x, \eta)$;

(ii) $\xi(t, x, \eta ; z) \geq r \Leftrightarrow z \notin \operatorname{re}(t, x, \eta)-\operatorname{int} C(t, x, \eta)$.

\section{Semicontinuity of Solution Mapping}

In this section, we discuss the upper and lower semicontinuity of the solution mapping $S(\cdot)$ as a set-valued mapping from the set $T$ to $X$.

Lemma 5. Let $\widehat{\psi}: T \rightrightarrows X$ be a set-valued mapping with nonempty values and let $\widehat{f}: T \times X \rightarrow \mathbb{R}$ be a function. Let $\widehat{S}: T \rightrightarrows X$ be defined by

$$
\widehat{S}(t):=\{x \in \widehat{\psi}(t): \widehat{f}(t, x) \geq 0\}
$$

and let $t_{0} \in \operatorname{dom} \widehat{S}$. Assume that

(i) $\widehat{\psi}$ is u.s.c and compact valued at $t_{0}$;

(ii) for any $x_{0} \in \widehat{\psi}\left(t_{0}\right)$ any nets $\left\{t_{\alpha}\right\}$ with $t_{\alpha} \rightarrow t_{0}$ and $\left\{x_{\alpha}\right\}$ with $x_{\alpha} \rightarrow x_{0}$, if $\widehat{f}\left(t_{0}, x_{0}\right)<0 \Rightarrow \exists \alpha_{0}$, s.t. $\widehat{f}\left(t_{\alpha_{0}}, x_{\alpha_{0}}\right)<0$.

Then $\widehat{S}(\cdot)$ is u.s.c at $t_{0}$.

Proof. Suppose that $\widehat{S}(\cdot)$ is not u.s.c at $t_{0}$. Then there exist an open set $V \subseteq X$ satisfying $\widehat{S}\left(t_{0}\right) \subseteq V$, nets $\left\{t_{\alpha}\right\}$ with $t_{\alpha} \rightarrow t_{0}$ and $x_{\alpha} \in \widehat{S}\left(t_{\alpha}\right)$, such that $x_{\alpha} \notin V, \forall \alpha$.

Since $x_{\alpha} \in \widehat{\psi}\left(t_{\alpha}\right)$ and $\widehat{\psi}(\cdot)$ is u.s.c with compact values at $t_{0}$, by Proposition 2(ii), there exist $x_{0} \in \widehat{\psi}\left(t_{0}\right)$ and a subnet $\left\{x_{\beta}\right\}$ of $\left\{x_{\alpha}\right\}$ such that $x_{\beta} \rightarrow x_{0}$. Since $x_{\alpha} \notin V$, $x_{\beta} \notin V$ and $x_{0} \notin V$. In particular, $x_{0} \notin \widehat{S}\left(t_{0}\right)$; that is, $\widehat{f}\left(t_{0}, x_{0}\right)<0$. By assumption (ii), there exists an index $\beta_{0}$ such that $\widehat{f}\left(t_{\beta_{0}}, x_{\beta_{0}}\right)<0$. This contradicts the above condition that $x_{\alpha} \in \widehat{S}\left(t_{\alpha}\right)$ for all $\alpha$.

The example is given to illustrate that assumption (ii) of Lemma 5 is essential. 
Example 6. Let $T=[0,1]$ and $X=\mathbb{R}$. For each $t \in T$, let $\widehat{\psi}(t)=[-1,2]$. Let $t_{0}=0$. For each $(t, x) \in T \times X$, we define

$$
\widehat{f}(t, x)= \begin{cases}-x(x-1), & \text { if } t=t_{0}, \\ -(t+x) \cdot(x-2), & \text { if } t \in T \backslash t_{0} .\end{cases}
$$

It is easy to check that assumption (i) is satisfied. One has $\widehat{S}\left(t_{0}\right)=[0,1], \widehat{S}(t)=[-t, 2], t \in(0,1]$. Hence $\widehat{S}(\cdot)$ is not u.s.c at $t_{0}$. It is equally clear that assumption (ii) is violated. Indeed, there exist $x_{0}=3 / 2$ and nets $\left\{t_{\alpha}\right\} \subset(0,1 / 2)$ with $t_{\alpha} \rightarrow t_{0}$ and $\left\{x_{\alpha}\right\} \subset(1,2)$ with $x_{\alpha} \rightarrow x_{0}$; one has $\widehat{f}\left(t_{0}, x_{0}\right)=$ $-x_{0}\left(x_{0}-1\right)=-(3 / 4)<0$. However, for all $\alpha$, one has $\widehat{f}\left(t_{\alpha}, x_{\alpha}\right)=-\left(t_{\alpha}+x_{\alpha}\right)\left(x_{\alpha}-2\right)>0$. Hence, assumption (ii) of Lemma 5 is essential.

Remark 7. Obviously, for each $x_{0} \in \widehat{\psi}\left(t_{0}\right)$, assumption (ii) can be ensured by the upper semicontinuity of the real function $\widehat{f}(\cdot, \cdot)$ at $\left(t_{0}, x_{0}\right)$. Therefore, we can get Corollary 8 . It is worth noting that Corollary 8 is just Lemma 4.1 of [21]. Therefor, Lemma 5 improves Lemma 4.1 of [21].

Corollary 8. Let $\widehat{\psi}: T \rightrightarrows X$ be a mapping with nonempty values and let $\widehat{f}: T \times X \rightarrow \mathbb{R}$ be a function. Let $\widehat{S}: T \rightrightarrows X$ be defined by

$$
\widehat{S}(t):=\{x \in \widehat{\psi}(t): \widehat{f}(t, x) \geq 0\}
$$

and let $t_{0} \in \operatorname{dom} \widehat{S}$. Assume that

(i) $\widehat{\psi}$ is u.s.c and compact valued at $t_{0}$;

(ii) for each $x_{0} \in \widehat{\psi}\left(t_{0}\right) \widehat{f}$ is u.s.c at $\left(t_{0}, x_{0}\right)$.

Then $\widehat{S}(\cdot)$ is u.s.c at $t_{0}$.

Lemma 9. Let $\widehat{\psi}: T \rightrightarrows X$ be a mapping with nonempty values and let $\widehat{f}: T \times X \rightarrow \mathbb{R}$ be afunction. Let $\widehat{S}: T \rightrightarrows X$ be defined by

$$
\widehat{S}(t):=\{x \in \widehat{\psi}(t): \widehat{f}(t, x) \geq 0\}
$$

and let $t_{0} \in \operatorname{dom} \widehat{S}$. Assume that

(i) $\widehat{\psi}$ is l.s.c at $t_{0}$;

(ii) for any $x_{0} \in \widehat{\psi}\left(t_{0}\right)$ any nets $\left\{t_{\alpha}\right\}$ with $t_{\alpha} \rightarrow t_{0}$ and $\left\{x_{\alpha}\right\}$ with $x_{\alpha} \rightarrow x_{0}$, if $\widehat{f}\left(t_{0}, x_{0}\right) \geq 0 \Rightarrow \exists \alpha_{0}$, s.t. $\widehat{f}\left(t_{\alpha_{0}}, x_{\alpha_{0}}\right) \geq 0$.

Then $\widehat{S}(\cdot)$ is l.s.c at $t_{0}$.

Proof. Suppose that $\widehat{S}(\cdot)$ is not l.s.c at $t_{0}$. Then, by Proposition 2(i), there exist a net $\left\{t_{\alpha}\right\}$ with $t_{\alpha} \rightarrow t_{0}$ and $x_{0} \in \widehat{S}\left(t_{0}\right)$ such that, for any $x_{\alpha} \in \widehat{S}\left(t_{\alpha}\right)$, we have $x_{\alpha} \nrightarrow x_{0}$.

From $x_{0} \in \widehat{S}\left(t_{0}\right)$, we have $x_{0} \in \widehat{\psi}\left(t_{0}\right)$. As $\widehat{\psi}(\cdot)$ is l.s.c at $t_{0}$, there exists $\bar{x}_{\alpha} \in \widehat{\psi}\left(t_{\alpha}\right)$ such that $\bar{x}_{\alpha} \rightarrow x_{0}$. By the above contradiction assumption, there must exist subnet $\left\{\bar{x}_{\beta}\right\} \subset$ $\left\{\bar{x}_{\alpha}\right\}$ such that $\forall \beta$ with $\bar{x}_{\beta} \notin \widehat{S}\left(t_{\beta}\right)$; that is,

$$
\widehat{f}\left(t_{\beta}, \bar{x}_{\beta}\right)<0 \text {. }
$$

Since $x_{0} \in \widehat{S}\left(t_{0}\right)$, we have $\widehat{f}\left(t_{0}, x_{0}\right) \geq 0$. By assumption (ii), there exists an index $\beta_{0}$ such that $\widehat{f}\left(t_{\beta_{0}}, \bar{x}_{\beta_{0}}\right) \geq 0$, which contradicts (7). Thus $\widehat{S}(\cdot)$ is l.s.c at $t_{0}$.

The following example is given to illustrate that assumption (ii) of Lemma 9 is essential.

Example 10. Let $T=[0,1], X=\mathbb{R}$. For each $t \in T$, let $\widehat{\psi}(t)=$ $[-1,1]$. Let $t_{0}=0$. For each $(t, x) \in T \times X$, we define

$$
\widehat{f}(t, x)= \begin{cases}x\left(x+\frac{1}{2}\right), & \text { if } t=t_{0}, \\ t x, & \text { if } t \in T \backslash t_{0} .\end{cases}
$$

It is easy to check that assumption (i) is satisfied. It follows from a direct computation that $\widehat{S}\left(t_{0}\right)=[-1,-(1 / 2)] \cup[0,1]$ and $\widehat{S}(t)=[0,1], t \in(0,1]$. Hence $\widehat{S}(\cdot)$ is not l.s.c at $t_{0}$. It is equally clear that assumption (ii) is violated. Indeed, there exist $x_{0}=-(1 / 2)$ and nets $\left\{t_{\alpha}\right\} \subset(0,1 / 2)$ with $t_{\alpha} \rightarrow t_{0}$ and $\left\{x_{\alpha}\right\} \subset(-1,0)$ with $x_{\alpha} \rightarrow x_{0}$; one has $\widehat{f}\left(t_{0}, x_{0}\right)=x_{0}\left(x_{0}+\right.$ $(1 / 2))=0$. However, for all $\alpha$, one has $\widehat{f}\left(t_{\alpha}, x_{\alpha}\right)=t_{\alpha} x_{\alpha}<0$. Thus, assumption (ii) of Lemma 5 is essential.

Remark 11. (i) In [21], Sach and Tuan used a key assumption $\widehat{H}_{1}$ or $\widehat{H}_{2}$, which includes the information on the solution set, to obtain the continuity of set-valued mapping $\widehat{S}(\cdot)$. The main advantage of assumption (ii) in Lemma 9 is that it does not require any information on solution set $\widehat{S}(t)$ for each $t \in T$.

(ii) Obviously, for each $x_{0} \in \widehat{\psi}\left(t_{0}\right)$, assumption (ii) can be ensured by the lower semicontinuity of the real function $\widehat{f}(\cdot, \cdot)$ at $\left(t_{0}, x_{0}\right)$.

Now we give an example for illustrating Lemma 9.

Example 12. Let $T=[0,1]$ and $X=\mathbb{R}$. For each $t \in T$, let $\widehat{\psi}(t)=[-1,2]$. Let $t_{0}=1$. For each $(t, x) \in T \times X$, we define

$$
\widehat{f}(t, x)= \begin{cases}2, & \text { if } t=t_{0}, x=1, \\ x-1, & \text { if } t=t_{0}, x \neq 1, \\ -(x-t)(x-2), & \text { if } t \in T \backslash t_{0} .\end{cases}
$$

It is easy to check that assumptions (i) and (ii) are satisfied. From a direct computation, we have $\widehat{S}\left(t_{0}\right)=[1,2]$ and $\widehat{S}(t)=$ $[t, 2], t \in(0,1]$. Hence $\widehat{S}(\cdot)$ is l.s.c at $t_{0}$. However, the real function $\widehat{f}(\cdot, \cdot)$ is not lower semicontinuity at $\left(t_{0}, x_{0}\right)$, where $x_{0}=1 \in \widehat{\psi}\left(t_{0}\right)$.

By Remark 11(ii), we can get the following corollary.

Corollary 13. Let $\widehat{\psi}: T \rightrightarrows X$ be a mapping with nonempty values and let $\widehat{f}: T \times X \rightarrow \mathbb{R}$ be a function. Let $\widehat{S}: T \rightrightarrows X$ be defined by

$$
\widehat{S}(t):=\{x \in \widehat{\psi}(t): \widehat{f}(t, x) \geq 0\}
$$

and let $t_{0} \in \operatorname{dom} \widehat{S}$. Assume that

(i) $\widehat{\psi}$ is l.s.c at $t_{0}$;

(ii) for each $x_{0} \in \widehat{\psi}\left(t_{0}\right) \widehat{f}$ is l.s.c at $\left(t_{0}, x_{0}\right)$. 
Then $\widehat{S}(\cdot)$ is l.s.c at $t_{0}$.

For each $(t, x) \in T \times X$, we set

$$
f(t, x)=\inf _{\eta \in A_{1}(t, x)} \inf _{z \in F(t, x, \eta)} \xi(t, x, \eta ; z) .
$$

Denote by $\psi(t)$ the fixed points of $A_{0}(t, \cdot)$ :

$$
\psi(t)=\left\{x \in X: x \in A_{0}(t, x)\right\} .
$$

In the sequel, we always assume that $t_{0} \in \operatorname{dom} S$.

As a direct consequence of Lemma 5 and Proposition 4, we can get the following results on the semicontinuity of $S(\cdot)$.

Theorem 14. Suppose the following conditions are satisfied:

(i) $\psi$ is u.s.c and compact valued at $t_{0}$;

(ii) for any $x_{0} \in \psi\left(t_{0}\right)$ any nets $\left\{t_{\alpha}\right\}$ with $t_{\alpha} \rightarrow t_{0}$ and $\left\{x_{\alpha}\right\}$ with $x_{\alpha} \rightarrow x_{0}$, if $f\left(t_{0}, x_{0}\right)<0 \Rightarrow \exists \alpha_{0}$, s.t. $f\left(t_{\alpha_{0}}, x_{\alpha_{0}}\right)<0$.

Then $S(\cdot)$ is u.s.c at $t_{0}$.

Proof. For each $t \in T$, we prove the following equation:

$$
S(t)=\{x \in \psi(t): f(t, x) \geq 0\} .
$$

First, we prove

$$
S(t) \subseteq\{x \in \psi(t): f(t, x) \geq 0\} .
$$

Indeed, for each $t \in T$ and $x \in S(t), x \in A_{0}(t, x)$ and for all $\eta \in A_{1}(t, x)$, we have

$$
F(t, x, \eta) \subset Y \backslash-\operatorname{int} C(t, x, \eta) .
$$

It implies that $\inf _{z \in F(t, x, \eta)} \xi(t, x, \eta ; z) \geq 0$ for all $\eta \in A_{1}(t, x)$ by Proposition 4(ii); that is, $f(t, x) \geq 0$. Since $\psi(t)=\{x \in X$ : $\left.x \in A_{0}(t, x)\right\}, x \in \psi(t)$. By the arbitrariness of $x \in S(t)$, we have $S(t) \subseteq\{x \in \psi(t): f(t, x) \geq 0\}$.

Let $A=\{x \in \psi(t): f(t, x) \geq 0\}$. For each $t \in T$, we need to prove $A \subseteq S(t)$. Indeed, for each $t \in T$ and $x \in A$, we have $x \in \psi(t)=\left\{x \in X: x \in A_{0}(t, x)\right\}$ and

$$
\inf _{\eta \in A_{1}(t, x)} \inf _{z \in F(t, x, \eta)} \xi(t, x, \eta ; z) \geq 0 .
$$

Thus $x \in A_{0}(t, x)$ and for all $\eta \in A_{1}(t, x)$, for any $z \in$ $F(t, x, \eta)$ such that $z \notin-\operatorname{int} C(t, x, \eta)$ by Proposition 4(ii), that is, $F(t, x, \eta) \subset Y \backslash-\operatorname{int} C(t, x, \eta), \forall \eta \in A_{1}(t, x)$. Thus, $x \in S(t)$ and (13) holds. By virtue of Lemma 5 and (13), we get that $S(\cdot)$ is u.s.c at $t_{0}$.

The following is given to illustrate that Theorem 14 holds, but Theorem 3.1 of [19] is not applicable.

Example 15. Let $T=[0,1], X=\mathbb{R}$, and $Y=\mathbb{R}^{2}$. For each $(t, x, \eta) \in T \times X \times X$, let $C(t, x, \eta)=\mathbb{R}_{+}^{2}$. For each $(t, x) \in T \times X$, $A_{1}(t, x)=[0,1]$. For each $t \in T$, let $\psi(t)=[-1,1]$. Let $t_{0}=0$. For each $(t, x, \eta) \in T \times X \times X$, we define

$$
F(t, x, \eta)= \begin{cases}{[-3,1] \times[0,1],} & \text { if } t=t_{0}, \\ {[x-t, x] \times[-1, y],} & \text { if } t \in T \backslash t_{0} .\end{cases}
$$

It is easy to check that assumptions (i)-(ii) are satisfied. It follows from a direct computation that $S\left(t_{0}\right)=[-1,1]$ and $S(t)=[t, 1]$, for each $t \in T$. Thus $S(\cdot)$ is u.s.c at $t_{0}$. However, for each $(x, \eta) \in \psi(t)=[-1,1] \times[0,1]$, the mapping $F(\cdot, x, \eta)$ is not $\mathbb{R}_{+}^{2}$-lower semicontinuity at $t_{0}$. Indeed, $(x, \eta) \in \psi(t)=$ $[-1,1] \times[0,1]$; there exists $z_{0}=(-3,0) \in F\left(t_{0}, x, \eta\right)$ and a neighborhood $U_{0}=(0,1 / 2) \times(0,1 / 2)$ of $0_{\mathbb{R}^{2}}$ such that for any neighborhood, $U\left(t_{0}\right)$ of $t_{0}$,

$$
F(t, x, \eta) \cap\left(z_{0}+U_{0}-\mathbb{R}_{+}^{2}\right)=\emptyset, \quad \forall t \in U\left(t_{0}\right) \cap T .
$$

Therefore, our result is applicable, but Theorem 3.1 of [19] is not applicable, since assumption (iii) in Theorem 3.1 of [19] does not hold.

Theorem 16. Suppose the following conditions are satisfied:

(i) $\psi$ is l.s.c at $t_{0}$;

(ii) for any $x_{0} \in \psi\left(t_{0}\right)$, any nets $\left\{t_{\alpha}\right\}$ with $t_{\alpha} \rightarrow t_{0}$ and $\left\{x_{\alpha}\right\}$ with $x_{\alpha} \rightarrow x_{0}$, if $f\left(t_{0}, x_{0}\right) \geq 0 \Rightarrow \exists \alpha_{0}$, s.t. $f\left(t_{\alpha_{0}}, x_{\alpha_{0}}\right) \geq 0$.

Then $S(\cdot)$ is l.s.c at $t_{0}$.

Proof. Similar to the proofs of Theorem 14, we have

$$
S(t)=\{x \in \psi(t): f(t, x) \geq 0\} .
$$

Therefore, by virtue of Lemma 9, we get that $S(\cdot)$ is l.s.c at $t_{0}$.

Remark 17. (i) In [20, 21], the authors used some key assumptions (assumption (ii) of Theorem 5.1 in [20] and assumption (ii) of Theorem 4.2 in [21]), which include information on the solution set, to obtain the lower semicontinuity of the solution mapping $S(\cdot)$. In $[16,18,19]$, the authors used some assumptions, which also include information on the solution set. The main advantage of assumption (ii) in Theorem 16 is that it does not require any information on solution set $S(t)$ for each $t \in T$.

(ii) The lower semicontinuity results on the problem (PGWKFI) in $[9,13]$ require the cone-strict monotonicity of the mapping F. However, Theorem 16 does not use the assumption.

(iii) The results of [21] must require that the mapping $F$ is bounded. However, Theorem 16 does not require the assumption.

(iv) In [17], Wang and Li used a key assumption $H$ to obtain the lower semicontinuity of the solution mapping $S(\cdot)$ of the problem (PGWKFI). Assumption (ii) of Theorem 16 is different from assumption $H$ in Theorem 3.3 of [17].

(v) In $[9,13,16-19]$, the authors used the assumption that $F(\cdot, \cdot, \cdot)$ is lower semicontinuity on $t_{0} \times X \times X$. However, Theorem 16 does not use the assumption.

Now we give an example to show cases (ii)-(v) of Remark 17.

Example 18. Let $T=[0,1], X=\mathbb{R}$, and $Y=\mathbb{R}^{2}$. For each $(t, x, \eta) \in T \times X \times X$, let $C(t, x, \eta)=\mathbb{R}_{+}^{2}$. For each $(t, x) \in T \times X$, 
$A_{1}(t, x)=[0,2]$. For each $t \in T$, let $\psi(t)=[-1,1]$. Let $t_{0}=0$. For each $(t, x, \eta) \in T \times X \times X$, we define

$$
F(t, x, \eta)= \begin{cases}\{\eta-x\} \times[-1,1], & \text { if } t=t_{0}, \\ {[-1, x+1] \times[1+\eta,+\infty),} & \text { if } t \in T \backslash t_{0} .\end{cases}
$$

It is easy to check that assumptions (i)-(ii) are satisfied. Obviously, $F$ is not l.s.c on $t_{0} \times X \times X$ and is also not bounded on $(0,1] \times X \times X$. It follows from a direct computation that $S\left(t_{0}\right)=[-1,0], S(t)=[-1,1]$, for each $t \in T \backslash\left\{t_{0}\right\}$. Thus $S(\cdot)$ is l.s.c at $t_{0}$.

However, for any $x \in \psi\left(t_{0}\right)=[-1,1], \eta \in A_{1}\left(t_{0}, x\right)=$ $[0,2]$ with $x \neq \eta$, we have

$$
\begin{aligned}
& F\left(t_{0}, x, \eta\right)+F\left(t_{0}, \eta, x\right) \\
& \quad=\{0\} \times[-2,2] \not \subset-\operatorname{int} C\left(t_{0}, x, \eta\right)=-\operatorname{int} \mathbb{R}_{+}^{2} ;
\end{aligned}
$$

that is, $F\left(t_{0}, \cdot, \cdot\right)$ is not cone-strictly monotone on $\psi\left(t_{0}\right) \times$ $\bigcup_{x \in \psi\left(t_{0}\right)} A_{1}\left(t_{0}, x\right)$. Therefore, Theorem 16 is applicable, but Theorem 4.1 of [9] and Theorem 3.1 of [13] are not applicable.

Since there exists $f_{0}=(1,1) \in \mathbb{R}_{+}^{2} \backslash\left\{0_{\mathbb{R}_{+}^{2}}\right\}$ with $\inf _{z \in F\left(t_{0}, x, \eta\right)}=\eta-x-1=0 \nRightarrow \eta=x$; that is, the assumption $H$ in Theorem 3.3 of [17] does not hold, Theorem 3.3 of [17] is also not applicable.

\section{Concluding Remarks}

In this paper, we have obtained the upper and lower semicontinuity of the solution mapping to a class of parametric generalized weak Ky Fan inequality. The novelty of the present paper consists in the following aspects.

(i) In order not to use the convexity of generalized convexity assumption, we adopt a nonlinear scalarization approach to obtain the solution mapping to problem (PGWKFI). To avoid using the assumption that the objective mapping $F$ is bounded or compact, we applied a nonlinear scalarization function defined in [24], which is different from ones in $[20,21]$.

(ii) In [16, 18-21], the authors used some key assumptions (including the information on the solution set) to get the upper and lower semicontinuity of the solution mapping of problem (PGWKFI). However, we also obtained the upper and lower semicontinuity of the solution mapping of problem (PGWKFI) under some new assumptions, which do not include any information on the solution set.

(iii) The idea of the present paper is also suitable to consider the semicontinuity of the solution mapping to the following parametric generalized weak Ky Fan inequality:

find a point $x \in X$ such that $x \in A_{0}(t, x)$ and, for all $\eta \in A_{1}(t, x)$,

$$
F(t, x, \eta) \cap Y \backslash-\operatorname{int} C(t, x, \eta) \neq \emptyset .
$$

\section{Conflict of Interests}

The author declares that there is no conflict of interests regarding the publication of this paper.

\section{Acknowledgments}

This research was supported by the Basic and Advanced Research Project of CQ CSTC (Grant no. cstc2014jcyjA00044) and the Foundation of Chongqing University of Posts and Telecommunications for Scholars with Doctorate (Grant numbers: A2014-15 and A2014-16).

\section{References}

[1] K. Fan, "Extensions of two fixed point theorems of F. E. Browder," Mathematische Zeitschrift, vol. 112, pp. 234-240, 1969.

[2] F. Giannessi, Ed., Vector Variational Inequalities and Vector Equilibria: Mathematical Theories, Kluwer Academic Publishers, Dordrecht, The Netherlands, 2000.

[3] Q. H. Ansari and F. Flores-Bazán, "Generalized vector quasiequilibrium problems with applications," Journal of Mathematical Analysis and Applications, vol. 277, no. 1, pp. 246-256, 2003.

[4] X. B. Li and S. J. Li, "Existence of solutions for generalized vector quasi-equilibrium problems," Optimization Letters, vol. 4, no. 1, pp. 17-28, 2010.

[5] I. Sadeqi and C. G. Alizadeh, "Existence of solutions of generalized vector equilibrium problems in reflexive Banach spaces," Nonlinear Analysis: Theory, Methods \& Applications, vol. 74, no. 6, pp. 2226-2234, 2011.

[6] L. Q. Anh and P. Q. Khanh, "Semicontinuity of the solution set of parametric multivalued vector quasiequilibrium problems," Journal of Mathematical Analysis and Applications, vol. 294, no. 2, pp. 699-711, 2004.

[7] L. Q. Anh and P. Q. Khanh, "Continuity of solution maps of parametric quasiequilibrium problems," Journal of Global Optimization, vol. 46, no. 2, pp. 247-259, 2010.

[8] Y. H. Cheng and D. L. Zhu, "Global stability results for the weak vector variational inequality," Journal of Global Optimization, vol. 32, no. 4, pp. 543-550, 2005.

[9] X. H. Gong, "Continuity of the solution set to parametric weak vector equilibrium problems," Journal of Optimization Theory and Applications, vol. 139, no. 1, pp. 35-46, 2008.

[10] X. H. Gong and J. C. Yao, "Lower semicontinuity of the set of efficient solutions for generalized systems," Journal of Optimization Theory and Applications, vol. 138, no. 2, pp. 197205, 2008.

[11] Y. Han and X. H. Gong, "Lower semicontinuity of solution mapping to parametric generalized strong vector equilibrium problems," Applied Mathematics Letters, vol. 28, pp. 38-41, 2014.

[12] N. J. Huang, J. Li, and H. B. Thompson, "Stability for parametric implicit vector equilibrium problems," Mathematical and Computer Modelling, vol. 43, no. 11-12, pp. 1267-1274, 2006.

[13] C. R. Chen, S. J. Li, and K. L. Teo, "Solution semicontinuity of parametric generalized vector equilibrium problems," Journal of Global Optimization, vol. 45, no. 2, pp. 309-318, 2009.

[14] S. J. Li, G. Y. Chen, and K. L. Teo, "On the stability of generalized vector quasivariational inequality problems," Journal of Optimization Theory and Applications, vol. 113, no. 2, pp. 283295, 2002.

[15] S.-J. Li, H.-M. Liu, and C.-R. Chen, "Lower semicontinuity of parametric generalized weak vector equilibrium problems," Bulletin of the Australian Mathematical Society, vol. 81, no. 1, pp. 85-95, 2010.

[16] S. J. Li and Z. M. Fang, "Lower semicontinuity of the solution mappings to a parametric generalized Ky Fan inequality," 
Journal of Optimization Theory and Applications, vol. 147, no. 3, pp. 507-515, 2010.

[17] Q. Wang and S. Li, "Lower semicontinuity of the solution mapping to a parametric generalized vector equilibrium problem," Journal of Industrial and Management Optimization, vol. 10, no. 4, pp. 1225-1234, 2014.

[18] Z. Y. Peng, X. M. Yang, and J. W. Peng, "On the lower semicontinuity of the solution mappings to parametric weak generalized Ky Fan inequality," Journal of Optimization Theory and Applications, vol. 152, no. 1, pp. 256-264, 2012.

[19] B. Chen and N.-J. Huang, "Continuity of the solution mapping to parametric generalized vector equilibrium problems," Journal of Global Optimization, vol. 56, no. 4, pp. 1515-1528, 2013.

[20] P. H. Sach, "New nonlinear scalarization functions and applications," Nonlinear Analysis: Theory, Methods \& Applications, vol. 75, no. 4, pp. 2281-2292, 2012.

[21] P. H. Sach and L. A. Tuan, "New scalarizing approach to the stability analysis in parametric generalized Ky Fan inequality problems," Journal of Optimization Theory and Applications, vol. 157, no. 2, pp. 347-364, 2013.

[22] P. H. Sach and N. B. Minh, "Continuity of solution mappings in some parametric non-weak vector Ky Fan inequalities," Journal of Global Optimization, vol. 57, no. 4, pp. 1401-1418, 2013.

[23] C. Berge, Topological Spaces, Oliver and Boyd, London, UK, 1963.

[24] G. Y. Chen, X. Q. Yang, and H. Yu, "A nonlinear scalarization function and generalized quasi-vector equilibrium problems," Journal of Global Optimization, vol. 32, no. 4, pp. 451-466, 2005.

[25] J. P. Aubin and I. Ekeland, Applied Nonlinear Analysis, Wiley, New York, NY, USA, 1984.

[26] F. Ferro, "A minimax theorem for vector-valued functions," Journal of Optimization Theory and Applications, vol. 60, no. 1, pp. 19-31, 1989. 


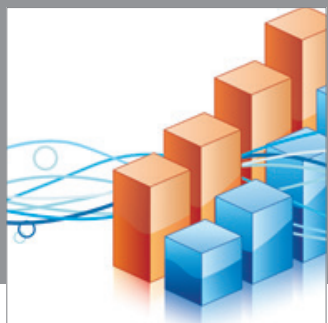

Advances in

Operations Research

mansans

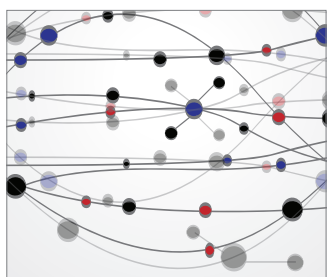

The Scientific World Journal
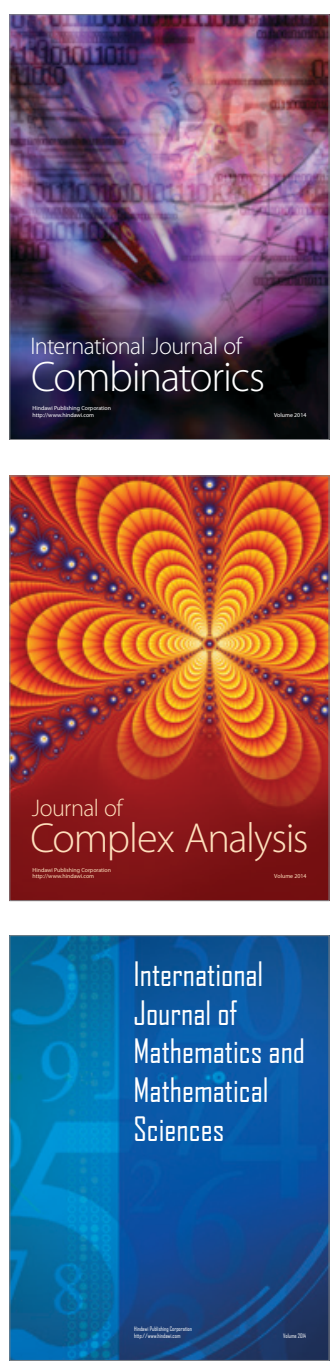
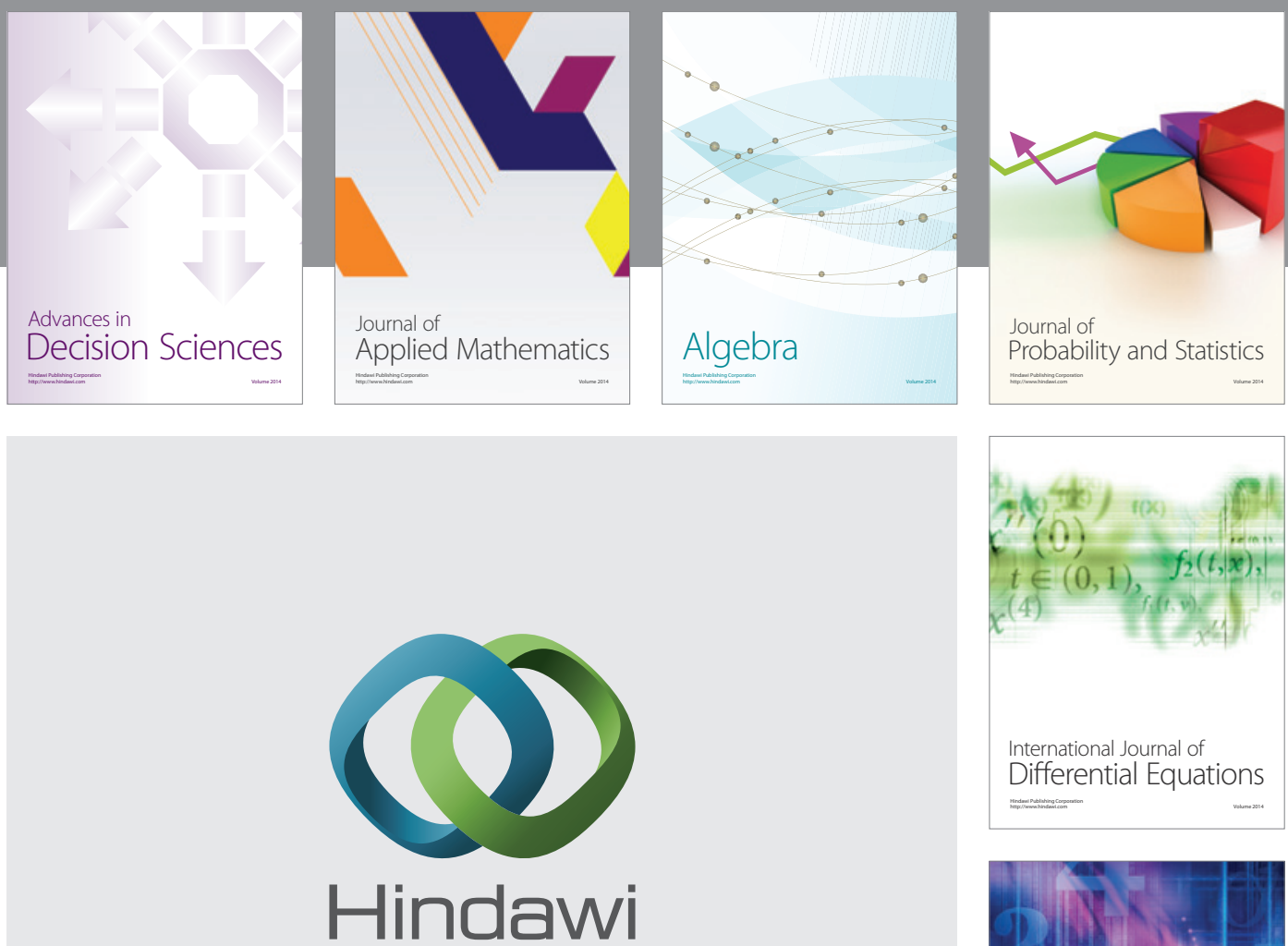

Submit your manuscripts at http://www.hindawi.com
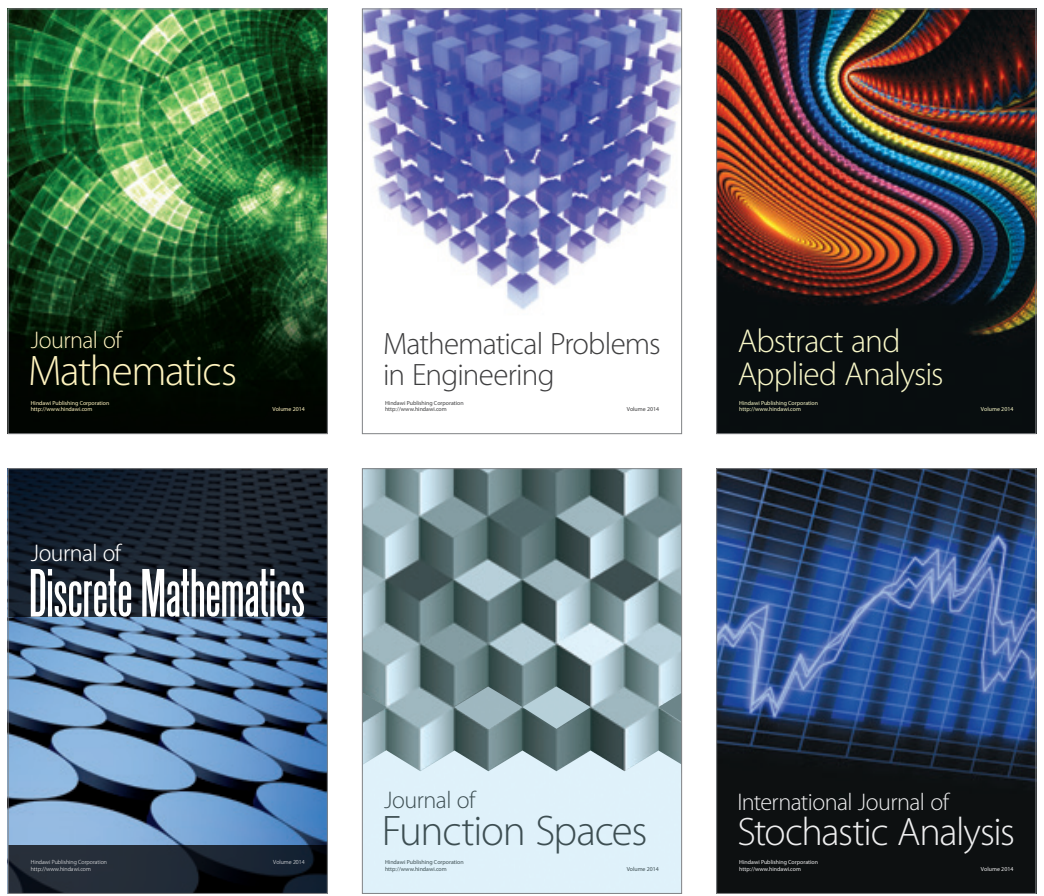

Journal of

Function Spaces

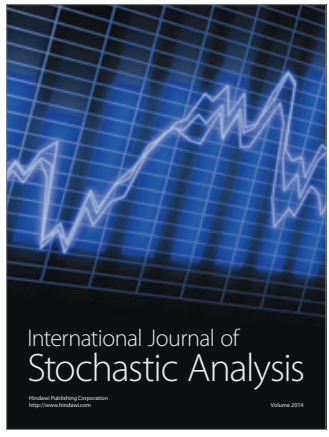

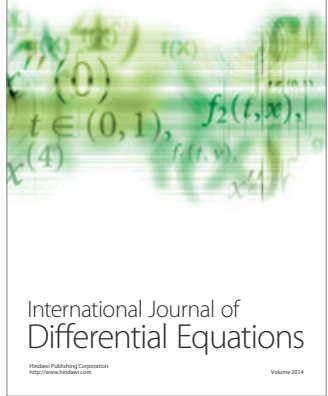
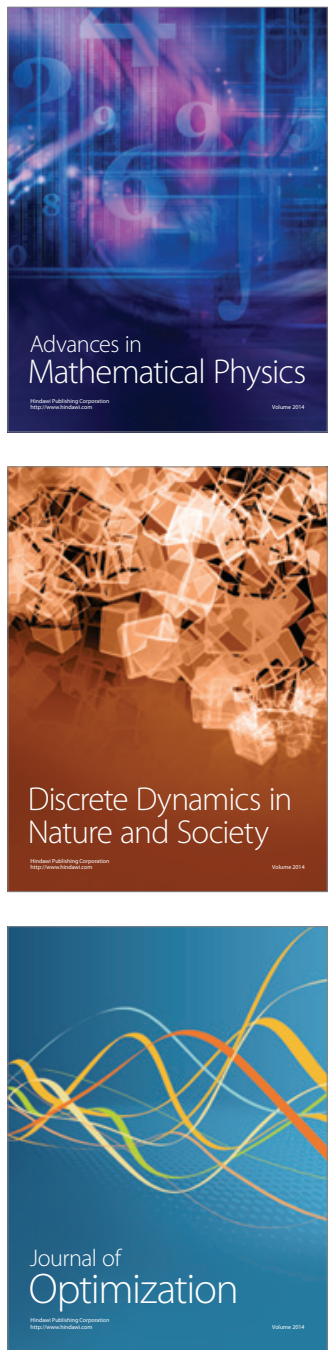